\title{
Prognostic impact of the pretreatment albumin to alkaline phosphatase ratio for nonmetastatic breast cancer patients
}

This article was published in the following Dove Press journal: Cancer Management and Research

\author{
Zhi-Qing Long ${ }^{1} * *$ \\ Xin Hua ${ }^{1, *}$ \\ Wen-Wen Zhang' \\ Shao-Wen Lv' \\ Jia-Peng Deng' \\ Ling Guo ${ }^{2}$ \\ Zhen-Yu He' \\ Huan-Xin Lin'
}

'Department of Radiotherapy, Sun Yatsen University Cancer Center,

Guangzhou, Guangdong, People's

Republic of China; ${ }^{2}$ Department of

Nasopharyngeal Carcinoma, Sun Yat-sen University Cancer Center, Guangzhou,

Guangdong, People's Republic of China

*These authors contributed equally to this work

Correspondence: Huan-Xin Lin; Zhen-Yu $\mathrm{He}$

Department of Radiotherapy, Sun Yat-sen University Cancer Center, No. 65I,

Dongfeng East Road, Guangzhou,

Guangdong 510060, People's Republic of

China

Tel +86 $2087343543 ;+862087343392$

Fax +862087343543; +862087343392

Email linhx@sysucc.org.cn;

hezhy@sysucc.org.cn
Introduction: Albumin and alkaline phosphatase have been previously demonstrated as independent prognostic factors for various types of cancer. Here, we aimed to explore the potential value of pretreatment albumin to alkaline phosphatase ratio (AAPR) on overall survival (OS) in nonmetastatic breast cancer patients.

Methods: A total of 746 nonmetastatic breast cancer patients were enrolled in this study. Receiver characteristic curve was used to analyze the AAPR threshold. Survival analysis was conducted using the Kaplan-Meier method and compared with the log-rank test. Both univariate and multivariate analyses were performed using Cox proportional hazards regression methodology.

Results: The optimal cutoff value of AAPR in predicting OS in nonmetastatic breast cancer patients was 0.525 . Increased pretreatment AAPR level was related to age at diagnosis $(\geq 60$ years vs $<60$ years, $P=0.000$ ), tumor size ( $\mathrm{T} \leq 2 \mathrm{~cm}$ vs $\mathrm{T}>2 \mathrm{~cm}, P=0.034)$, estrogen receptor (positive vs negative, $P=0.022$ ), progesterone receptor (positive vs negative, $P=0.025$ ), carcino-embryonic antigen (abnormal vs normal, $P=0.016$ ), surgery (lumpectomy vs mastectomy, $P=0.002$ ), chemotherapy (yes vs no, $P=0.004$ ), radiotherapy (yes vs no, $P=0.013$ ), endocrine therapy (yes vs no, $P=0.027$ ) but not with lymph node involvement, HER-2 status or CA-153. The 5 -year OS rate was $80.16 \%$ for the low AAPR group and $92.66 \%$ for the high AAPR group. Kaplan-Meier analysis indicated that patients with low-AAPR levels had shorter OS than patients with high-AAPR levels $(P=0.001)$. N classification $(P<0.05), \mathrm{Ki}-67$ $(\mathrm{HR}=3.603,95 \% \mathrm{CI}=1.046-12.414, P=0.042)$ and AAPR $(\mathrm{HR}=0.447,95 \% \mathrm{CI}=0.205-0.976$, $P=0.043$ ) were related to OS in multivariate analyses, respectively.

Conclusion: AAPR is an independent prognostic factor for OS in nonmetastatic breast cancer patients. Further prospective studies are required to confirm our findings.

Keywords: nonmetastatic breast cancer, prognosis, pretreatment albumin, alkaline phosphatase

\section{Introduction}

Breast cancer is one of the most malignant tumors to that poses a significant threat to female health. ${ }^{1}$ Despite a number of effective treatments including surgery, chemotherapy, radiotherapy, endocrine therapy, and targeted therapy, it remains the second most prevalent lethal cancer in women all over the world. ${ }^{2}$ Currently, tumor size, lymph node status, and metastasis are conventionally described as prognostic factors. Furthermore, estrogen receptor (ER), progesterone receptor (PR), and human epidermal growth factor receptor 2 (HER-2) status are broadly applied in clinic to recognize the different molecular subtypes of breast cancer for 
accurate treatment. However, a simpler and more economic index to assess the clinical outcomes for early breast patients effectively is required.

Recently, inflammation and immunity are research hotspots in cancer. ${ }^{3}$ Various serum pretreatment markers have been analyzed to assess whether they offer more valuable information in diagnosis and prognosis in breast cancer. These include neutrophil-to-lymphocyte ratio (NLR), monocyte-to-lymphocyte ratio, ${ }^{4}$ and pretreatment prognostic nutritional index (PNI). ${ }^{5}$ Serum albumin (ALB), produced by the liver, reflects body nutritional status and general physiological function by maintaining nitrogen balance, accelerating damage repair, and reducing inflammation. ${ }^{6}$ In addition, ALP, a hydrolase that participates in epithelial-mesenchymal transition (EMT) ${ }^{7}$ and ERK1/2 dephosphorylation and other bioprocesses, ${ }^{8}$ has been confirmed to be elevated and related to poor prognosis in multiple cancers, such as gastric cancer and prostate cancer. ${ }^{9,10}$

The AAPR is defined as the serum ALB level divided by the serum ALP level. The AAPR was first used as a prognostic index for hepatocellular carcinoma in 2015. ${ }^{11}$ In addition, AAPR has been trialled as a novel prognostic factor of OS for metastatic nasopharyngeal carcinoma and pancreatic ductal adenocarcinoma. $^{12,13}$ However, the prognostic capability of the pretreatment AAPR value for early breast cancer patients still remains unknown. Thus, we examined the role of the AAPR for nonmetastatic breast cancer patients undergoing surgery and its relation with other clinical features.

\section{Methods}

\section{Patients selection}

We retrospectively reviewed female breast cancer patients who received surgical treatment in Sun Yat-Sen University Cancer Center (Guangzhou, People's Republic of China) from November 2011 to February 2013. The exclusion criteria were: 1) incomplete baseline data; 2) no surgery; 3) metastatic breast cancer patients; 4) synchronal malignancies; 5) ductal carcinoma in situ. All subjects enrolled provided their written informed consent and the clinic ethics committee approval was obtained from Sun Yat-sen University Cancer Center (Guangzhou, People's Republic of China). This study was conducted in accordance with the Declaration of Helsinki.

\section{Data collection}

All patient information was intensively reviewed and obtained from the hospital database. The overall survival
(OS) time was defined as the period from surgery to death or to the last follow-up date. Pathological staging was assessed using the TNM staging system of the AJCC 7th edition via both postoperative histopathological inspection and clinical assessment. According to pathological reports, we identified ER and PR positive if there were at least $1 \%$ positive heterologous tumor cell nuclei in the sample evaluated by immunohistochemistry (IHC). HER-2 status was assessed using a semiquantitative score $(0-3+)$. Patients with $2+$ IHC staining for HER2 underwent fluorescence in-situ hybridization(Fish) to confirm HER2 positivity or negativity. AAPR was calculated as follows: AAPR = pretreatment serum albumin level $(\mathrm{g} / \mathrm{L}) /$ alkaline phosphatase level(U/L).

\section{Statistical analysis}

The optimal cutoff value of the AAPR was determined by the receiver operating characteristic (ROC) curve analysis of OS for early breast cancer patients. Baseline characteristics were compared using Fisher's exact test or Chisquared test. The Kaplan-Meier method was used for survival analyses and compared using the log-rank test. Univariate and multivariate analyses (Cox proportional hazards regression model) were conducted to assess the effect of potential confounding factors on OS. $P<0.05$ was considered statistically significant. All statistical analyses were processed using Graphpad Prism 6 (GraphPad Software Inc., La Jolla, CA, USA) and SPSS version 23.0 (SPSS, Inc., Chicago, IL) in this study.

\section{Results}

\section{Patient clinic characteristics}

We incorporated a total of 746 consecutive patients with surgery and histopathologically diagnosed early breast cancer in SYSUCC. The baseline characteristics of the patients are shown in Table 1. Pathological $\mathrm{T}$ stage was 301, 368, 39, 38 patients for T1, T2, T3 and T4 stage patients, respectively. There are $569(76.3 \%)$ patients having invasive ductal carcinoma. Among 746 patients, there are $199(26.7 \%)$ patients in stage I, $364(48.8 \%)$ patients in stage II, $183(24.5 \%)$ patients in stage III. ERstatus varied from positive $(\mathrm{n}=522,70.0 \%)$, negative $(\mathrm{n}=203,27.2 \%)$ to unknown ( $\mathrm{n}=21,2.8 \%)$. About $468(62.7 \%)$ of all enrollees are positive for PRstatus. According to the ROC results, patients were divided into a AAPR-low group (AAPR $<0.525, \mathrm{n}=125,16.8 \%$ ) and a AAPR-high group (AAPR $\geq 0.525, \mathrm{n}=621,83.2 \%$ ). 
Table I Basic characteristics of the enrolled patients

\begin{tabular}{|c|c|c|}
\hline Characteristic & Group & $\mathbf{N}(\%)$ \\
\hline \multirow[t]{2}{*}{ Age } & $\geq 60$ & $127(17.0)$ \\
\hline & $<60$ & $619(83.0)$ \\
\hline \multirow[t]{2}{*}{ Histopathological type } & Invasive ducal carcinoma & $569(76.3)$ \\
\hline & Others & $177(23.7)$ \\
\hline \multirow[t]{4}{*}{ T Classification } & TI & $301(40.3)$ \\
\hline & $\mathrm{T} 2$ & $368(49.3)$ \\
\hline & T3 & $39(5.2)$ \\
\hline & $\mathrm{T} 4$ & $38(5.1)$ \\
\hline \multirow[t]{4}{*}{ N Classification } & No & $407(54.6)$ \\
\hline & NI & $182(24.4)$ \\
\hline & N2 & $86(11.5)$ \\
\hline & N3 & $71(9.5)$ \\
\hline \multirow{3}{*}{ Clinial stage } & 1 & $199(26.7)$ \\
\hline & II & $364(48.8)$ \\
\hline & III & $183(24.5)$ \\
\hline \multirow[t]{4}{*}{ Histological grade } & GI & $20(2.7)$ \\
\hline & G2 & $480(64.3)$ \\
\hline & G3 & $146(19.6)$ \\
\hline & Unknown & $100(13.4)$ \\
\hline \multirow[t]{3}{*}{ Estrogen receptor } & Positive & $522(70.0)$ \\
\hline & Negative & $203(27.2)$ \\
\hline & Unknown & $21(2.8)$ \\
\hline Progesterone & Positive & $468(62.7)$ \\
\hline \multirow[t]{2}{*}{ receptor } & Negative & $256(34.8)$ \\
\hline & Unknown & $22(2.9)$ \\
\hline Human epidermal & Positive & $219(29.4)$ \\
\hline growth factor receptor & Negative & $421(56.4)$ \\
\hline type 2 (HER-2) & Unknown & $106(14.2)$ \\
\hline \multirow[t]{3}{*}{ CEA } & Normal & $646(86.6)$ \\
\hline & Abnormal & $67(9.0)$ \\
\hline & Unknown & $33(4.4)$ \\
\hline \multirow[t]{3}{*}{ CA-I53 } & Normal & $630(84.5)$ \\
\hline & Abnormal & $81(10.9)$ \\
\hline & Unknown & $35(4.7)$ \\
\hline \multirow[t]{3}{*}{ Ki-67 } & $\geq 14$ & $463(62.1)$ \\
\hline & $<14$ & $243(32.6)$ \\
\hline & Unknown & $40(5.4)$ \\
\hline \multirow[t]{2}{*}{ Surgery } & Mastectomy & $570(76.4)$ \\
\hline & Lumpectomy & $176(23.6)$ \\
\hline \multirow[t]{2}{*}{ Chemotherapy } & Yes & $589(79.0)$ \\
\hline & No & $157(2 \mid .0)$ \\
\hline \multirow[t]{2}{*}{ Radiotherapy } & Yes & $212(28.4)$ \\
\hline & No & 534 (7I.6) \\
\hline \multirow[t]{2}{*}{ Endocrine therapy } & Yes & $384(5 । .5)$ \\
\hline & No & $362(48.5)$ \\
\hline \multirow[t]{2}{*}{ AAPR } & $<0.525$ & $125(16.8)$ \\
\hline & $\geq 0.525$ & 621 (83.2) \\
\hline
\end{tabular}

Note: Clinical stage was according to the 7th edition of the American Joint Commission on Cancer staging system.

Abbreviations: ER, estrogen receptor; PR, progesterone receptor; HER-2, human epidermal growth factor receptor type 2; CEA, carcino-embryonic antigen; CAI5-3, cancer antigen 15-3; AAPR, pretreatment albumin to alkaline phosphatase ratio.

\section{Relationship between AAPR and patient characteristics}

The clinicopathological features for each group are shown in Table 2.

\section{Prognostic value of AAPR for OS}

The 5-year OS rate was $80.16 \%$ and $92.66 \%$ for the low and high AAPR groups, respectively. The Kaplan-Meier curve showed that patients in the low-AAPR group had poorer OS survival rate than those in the high-AAPR group $(P=0.001)$ (Figure 1).

In our study, based on univariate analysis, T classification, $\mathrm{N}$ classification, Ki-67, AAPR, ER, PR and HER-2 were important predictors of OS (all $P<0.05$ ). An AAPR $<0.525$ was statistically associated with inferior survival $\quad(\mathrm{HR}=0.363 ; 95 \% \quad \mathrm{CI}=0.191-0.689 ; \quad P=0.001)$. Neither the age nor the histopathological grade demonstrated any prognostic impact. In the Cox multivariate analysis model, the AAPR $\quad(\mathrm{HR}=0.447, \quad 95 \%$ $\mathrm{CI}=0.205-0.976, P=0.043)$ proved to be an independent prognostic factor of OS. Meanwhile, $\mathrm{N}$ classification and Ki-67 were independent prognostic factors (all $P<0.05$ ) (Table 3).

\section{Discussion}

In our study, we demonstrated that the AAPR was significantly related to the risk index of nonmetastatic breast cancer, including age, tumor size, ER, PR and carcino-embryonic antigen (CEA). The OS rate of the high AAPR group was significantly higher than that of the low AAPR group. Our findings were in accordance with previous studies of other type of cancers. ${ }^{11,12,14}$ The multivariate Cox proportional hazards regression model confirmed that the elevated pretreatment serum level of AAPR can independently predict OS for early breast cancer patients. To our knowledge, this is the first time that AAPR has been reported as an independent predictor of OS for breast cancer patients.

Tumor-related immune responses and nutritional assumption are associated with tumor development and progression. ${ }^{15}$ First, tumor growth, invasion and metastasis all require nutrition and the induction of an immunological response. Tumor cells can consume nutrients leading to cachexia. Then, to resist and kill cancer cells, the body may expend more nutrients and produce numerous inflammatory cytokines, such as tumor necrosis factor- $\alpha$ and vascular endothelial growth factor. ${ }^{16,17}$ The types of 
Table 2 Association between AAPR and clinicopathological factors in patients with nonmetastatic breast cancer

\begin{tabular}{|c|c|c|c|c|}
\hline \multirow[t]{2}{*}{ Variable } & \multirow[t]{2}{*}{ Total } & \multicolumn{2}{|c|}{ AAPR value } & \multirow[t]{2}{*}{$P$} \\
\hline & & Low & High & \\
\hline \multicolumn{5}{|l|}{ Age (year) } \\
\hline$<60$ & 127 & 45 & 82 & 0.000 \\
\hline$\geq 60$ & 619 & 80 & 539 & \\
\hline \multicolumn{5}{|l|}{ Tumor size } \\
\hline $\mathrm{T} \leq 2 \mathrm{~cm}$ & 302 & 41 & 261 & 0.034 \\
\hline $\mathrm{T}>2 \mathrm{~cm}$ & 444 & 84 & 360 & \\
\hline \multicolumn{5}{|l|}{$\mathbf{N}$} \\
\hline Positive & 339 & 59 & 280 & 0.369 \\
\hline Negative & 407 & 66 & 341 & \\
\hline \multicolumn{5}{|l|}{ ER } \\
\hline Positive & 522 & 76 & 446 & 0.022 \\
\hline Negative & 203 & 43 & 160 & \\
\hline \multicolumn{5}{|l|}{ PR } \\
\hline Positive & 468 & 67 & 401 & 0.025 \\
\hline Negative & 256 & 52 & 204 & \\
\hline \multicolumn{5}{|l|}{ HER-2 } \\
\hline Positive & 219 & 41 & 178 & 0.117 \\
\hline Negative & 421 & 62 & 359 & \\
\hline \multicolumn{5}{|l|}{ CEA } \\
\hline Abnormal & 67 & 18 & 49 & 0.016 \\
\hline Normal & 646 & 99 & 547 & \\
\hline \multicolumn{5}{|l|}{ CA- 153} \\
\hline Abnormal & 81 & 17 & 64 & 0.156 \\
\hline Normal & 630 & 100 & 530 & \\
\hline \multicolumn{5}{|l|}{ Surgery } \\
\hline Lumpectomy & 176 & 17 & 159 & 0.002 \\
\hline Mastectomy & 570 & 108 & 462 & \\
\hline \multicolumn{5}{|l|}{ Chemotherapy } \\
\hline Yes & 589 & 87 & 502 & 0.004 \\
\hline No & 157 & 38 & 119 & \\
\hline \multicolumn{5}{|l|}{ Radiotherapy } \\
\hline Yes & 212 & 25 & 187 & 0.013 \\
\hline No & 534 & 100 & 434 & \\
\hline \multicolumn{5}{|l|}{ Endocrine therapy } \\
\hline Yes & 384 & 54 & 330 & 0.027 \\
\hline No & 362 & 71 & 291 & \\
\hline
\end{tabular}

Note: Significant $P$-values are shown in bold.

Abbreviation: AAPR, pretreatment albumin to alkalinephosphatase ratio.

nutritional and inflammatory parameters have been reported to predict prognosis in breast cancer, including the pretreatment NLR, platelet to lymphocyte ratio, ${ }^{18}$ and PNI. Albumin and alkaline phosphatase are more direct

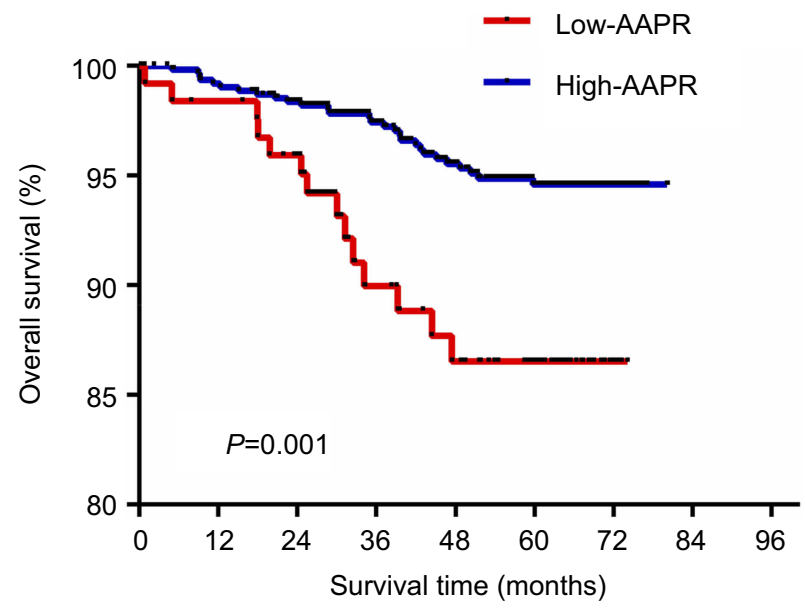

Figure I Overall survival based on the AAPR cutoff point of 0.525 in nonmetastatic breast cancer patients.

serum biochemical factors that reflect nutritional status of patients. Some abnormal conditions can affect the level of serum albumin, such as alcohol abuse, trauma, liver disease and hypermetabolism. ${ }^{19}$ Hypoalbuminemia may suppress immune responses, affecting both granuloma formation and macrophage activation. Consequently, some studies have demonstrated that a pretreatment ALB decrease is associated with poor outcome in several cancers due to malnutrition and an imbalanced tumor microenvironment, including renal cell carcinoma and colorectal cancer. $^{20,21}$ The level of ALP may be associated with bile duct obstruction, bone disease, hepatitis and malnutrition. Jaundice typically appears when ALP increases. According to previous studies, evaluation of ALP might manifest as a heavy tumor burden and thus relate to a poorer prognosis in esophageal carcinoma, colorectal cancer, and nasopharyngeal carcinoma. ${ }^{22-24}$

AAPR, obtained from the combination of albumin and alkaline phosphatase, may reflect nutritional state, and inflammatory and immune responses in cancer patients. Due to cancer development and progression, the body assumes an abnormal physiological status, such as nutritional decline and inflammatory activation, thus ultimately resulting in poor survival. Nei et al reported that AAPR may serve as a novel prognostic factor of OS for patients with metastatic nasopharyngeal carcinoma and consequently received cisplatin-based chemotherapy. ${ }^{12}$ Besides, a prior study indicated that AAPR was a useful prognostic factor for advanced HCC patients treated with nonstandard anti-cancer regimens. ${ }^{14}$ It should be noted that the optimal cutoff values of the AAPR are not the same for different cancers. 
Table 3 Survival analyses of clinicopathological factors and AAPR

\begin{tabular}{|c|c|c|c|c|}
\hline \multirow[t]{2}{*}{ Variable } & \multicolumn{2}{|l|}{ Univariate analysis } & \multicolumn{2}{|c|}{ Multivariate analysis } \\
\hline & HR (95\% Cl)) & $P$ & HR (95\% Cl) & $P$ \\
\hline Age & $1.412(0.676-2.95 I)$ & 0.359 & - & - \\
\hline \multicolumn{5}{|l|}{ T Classification } \\
\hline TI & \multicolumn{2}{|l|}{ I (reference) } & \multicolumn{2}{|l|}{ I (reference) } \\
\hline $\mathrm{T} 2$ & $2.74 \mid(I .24 I-6.053)$ & 0.013 & $2.152(0.789-5.868)$ & 0.134 \\
\hline T3 & $4.415(1.329-14.665)$ & 0.015 & $2.397(0.533-10.775)$ & 0.254 \\
\hline T4 & $4.617(1.390-15.338)$ & 0.013 & $1.715(0.296-9.918)$ & 0.547 \\
\hline \multicolumn{5}{|l|}{ N Classification } \\
\hline No & \multicolumn{2}{|l|}{ I (reference) } & \multicolumn{2}{|l|}{ I (reference) } \\
\hline NI & 3.207 (1.290-7.974) & 0.012 & $2.290(0.785-6.683)$ & 0.130 \\
\hline N2 & $6.019(2.231-15.607)$ & 0.000 & $4.405(1.442-13.453)$ & 0.009 \\
\hline N3 & II.742 (4.924-28.003) & 0.000 & $7.780(2.670-22.670)$ & 0.000 \\
\hline Ki-67 & 3.712 (I.452-9.492) & 0.006 & $3.603(1.046-12.4 \mid 4)$ & 0.042 \\
\hline APPR & $0.363(0.191-0.689)$ & 0.001 & $0.447(0.205-0.976)$ & 0.043 \\
\hline ER & $0.478(0.255-0.896)$ & 0.021 & 0.621 (0.194-1.992) & 0.423 \\
\hline PR & $0.467(0.25 I-0.869)$ & 0.016 & $1.149(0.365-3.614)$ & 0.831 \\
\hline HER-2 & $2.48 \mid(|.26|-4.883)$ & 0.009 & $1.286(0.580-2.85 \mathrm{I})$ & 0.536 \\
\hline Histological grade & $0.757(0.334-1.7 \mid 4)$ & 0.504 & $0.604(0.254-1.438)$ & 0.255 \\
\hline CEA & $2.165(0.960-4.885)$ & 0.063 & $0.615(0.20 \mathrm{I}-\mathrm{I} .88 \mathrm{I})$ & 0.394 \\
\hline Surgery & $6.135(1.526-26.128)$ & 0.011 & - & - \\
\hline Chemotherapy & $1.362(0.573-3.235)$ & 0.484 & - & - \\
\hline Radiotherapy & $1.272(0.677-2.392)$ & 0.455 & - & - \\
\hline Endocrine therapy & $0.361(0.190-0.688)$ & 0.002 & - & - \\
\hline
\end{tabular}

Note: Significant $P$-values are shown in bold.

Abbreviation: AAPR, pretreatment albumin to alkalinephosphatase ratio.

In our study, we initially investigated the relationship between AAPR and other clinical factors in nonmetastatic breast cancer patients and found that age, tumor size, ER, PR and CEA were related. A low-AAPR level was associated with poor OS in early breast cancer patients. A low-AAPR level may reflect the decline in both immunological function and anti-cancer ability. Furthermore, we first considered that pretreatment AAPR may independently and effectively predict OS in early breast cancer patients. As a low-cost, simple, accessible and non-invasive index, the AAPR could provide more information for clinicians and how best to treat. For example, low-AAPR value is associated with a poorer outcome in early breast cancer patients, therefore patients need to adequately increase their nutritional intake and receive proportionately more anti-inflammatory therapy according to clinical guidance. Immunotherapeutic drugs such as PD-1 inhibitors could provide a good therapeutic response in these patients. ${ }^{25}$ Furthermore, those patients with low-AAPR status should have their cancer status and physical condition reviewed more carefully compared with the high-AAPR group.
The limitations of our study is that it is a retrospective analysis of a single-center design, leading to selection bias and a relatively small sample size. In addition, we focused on the pretreatment serum index but failed to analyze the dynamic changes in the AAPR value during the whole process. Finally, biological mechanisms between AAPR and inflammation remain unclear. Therefore, further largescale prospective clinical trials and basic research studies are required to elucidate the molecular mechanisms of a low-AAPR value and poorer prognosis in patients with breast cancer.

In summary, we confirmed that the AAPR was related to OS of early breast cancer patients treated with surgery. A low-AAPR predicted poorer prognosis compared to the high-AAPR group. The AAPR was considered as an independent risk parameter in patients with nonmetastatic breast cancer. We provide more information for clinicians to determine treatment regimens through an easily accessible parameter of AAPR. 


\section{Acknowledgments}

This work was partly supported by the National Natural Science Foundation of China $(81772877,81773103$, and 81572848) and the Science and Technology Department of Guangdong Province, China (2017A030310422).

\section{Disclosure}

The authors report no conflicts of interest in this work.

\section{References}

1. DeSantis CE, Ma J, Goding Sauer A, Newman LA, Jemal A. Breast cancer statistics, 2017, racial disparity in mortality by state. $C A$ Cancer J Clin. 2017;67(6):439-448.

2. Ferlay J, Soerjomataram I, Dikshit R, et al. Cancer incidence and mortality worldwide: sources, methods and major patterns in GLOBOCAN 2012. Int $J$ Cancer. 2015;136(5):E359-386. doi:10.1002/ijc.29210

3. Topalian SL, Taube JM, Anders RA, Pardoll DM. Mechanism-driven biomarkers to guide immune checkpoint blockade in cancer therapy. Nat Rev Cancer. 2016;16(5):275-287. doi:10.1038/nrc.2016.36

4. Yamanouchi K, Kuba S, Sakimura C, et al. The relationship between peripheral neuropathy induced by docetaxel and systemic inflammation-based parameters in patients with breast cancer. Anticancer Res. 2017;37(12):6947-6951.

5. Mohri T, Mohri Y, Shigemori T, Takeuchi K, Itoh Y, Kato T. Impact of prognostic nutritional index on long-term outcomes in patients with breast cancer. World J Surg Oncol. 2016;14(1):170. doi:10.1186/s12957-016-0920-7

6. Don BR, Kaysen G. Serum albumin: relationship to inflammation and nutrition. Semin Dial. 2004;17(6):432-437. doi:10.1111/j.08940959.2004.17603.x

7. Rao SR, Snaith AE, Marino D, et al. Tumour-derived alkaline phosphatase regulates tumour growth, epithelial plasticity and disease-free survival in metastatic prostate cancer. $B r J$ Cancer. 2017;116 (2):227-236. doi:10.1038/bjc.2016.402

8. Sharma U, Pal D, Prasad R. A novel role of alkaline phosphatase in the ERK1/2 dephosphorylation in renal cell carcinoma cell lines: a new plausible therapeutic target. Biochimie. 2014;107(Pt B):406-409.

9. Namikawa T, Ishida N, Tsuda S, et al. Prognostic significance of serum alkaline phosphatase and lactate dehydrogenase levels in patients with unresectable advanced gastric cancer. Gastric Cancer. 2018. doi:10.1007/s10120-018-0897-8

10. Li D, Lv H, Hao X, Hu B, Song Y. Prognostic value of serum alkaline phosphatase in the survival of prostate cancer: evidence from a meta-analysis. Cancer Manag Res. 2018;10:3125-3139. doi:10.2147/ CMAR.S174237

11. Chan AW, Chan SL, Mo FK, et al. Albumin-to-alkaline phosphatase ratio: a novel prognostic index for hepatocellular carcinoma. Dis Markers. 2015;2015:564057. doi:10.1155/2015/105358

Cancer Management and Research

\section{Publish your work in this journal}

Cancer Management and Research is an international, peer-reviewed open access journal focusing on cancer research and the optimal use of preventative and integrated treatment interventions to achieve improved outcomes, enhanced survival and quality of life for the cancer patient.
12. Nie M, Sun P, Chen C, et al. Albumin-to-alkaline phosphatase ratio: a novel prognostic index of overall survival in cisplatin-based chemotherapy-treated patients with metastatic nasopharyngeal carcinoma. $J$ Cancer. 2017;8(5):809-815. doi:10. $7150 /$ jca. 17536

13. $\mathrm{Pu} \mathrm{N}, \mathrm{Gao} \mathrm{S}, \mathrm{Xu} \mathrm{Y}$, et al. Alkaline phosphatase-to-albumin ratio as a prognostic indicator in pancreatic ductal adenocarcinoma after curative resection. $J$ Cancer. 2017;8(16):3362-3370. doi:10.7150/ jca.20917

14. Cai X, Chen Z, Chen J, et al. Albumin-to-alkaline phosphatase ratio as an independent prognostic factor for overall survival of advanced hepatocellular carcinoma patients without receiving standard anti-cancer therapies. J Cancer. 2018;9(1):189-197. doi:10.7150/ jca.21799

15. Alwarawrah Y, Kiernan K, MacIver NJ. Changes in nutritional status impact immune cell metabolism and function. Front Immunol. 2018;9:1055. doi:10.3389/fimmu.2018.01055

16. Patel HJ, Patel BM. TNF-alpha and cancer cachexia: molecular insights and clinical implications. Life Sci. 2017;170:56-63. doi:10.1016/j.lfs.2016.11.033

17. Tang X, Yang Y, Yuan H, You J, Burkatovskaya M, Amar S. Novel transcriptional regulation of VEGF in inflammatory processes. $J$ Cell Mol Med. 2013;17(3):386-397. doi:10.1111/jcmm.12020

18. Krenn-Pilko S, Langsenlehner U, Thurner EM, et al. The elevated pretreatment platelet-to-lymphocyte ratio predicts poor prognosis in breast cancer patients. Br J Cancer. 2014;110(10):2524-2530. doi:10.1038/bjc.2014.163

19. Vazeille C, Jouinot A, Durand JP, et al. Relation between hypermetabolism, cachexia, and survival in cancer patients: a prospective study in 390 cancer patients before initiation of anticancer therapy. Am J Clin Nutr. 2017;105(5):1139-1147. doi:10.3945/ajen.116.137968

20. Stenman M, Laurell A, Lindskog M. Prognostic significance of serum albumin in patients with metastatic renal cell carcinoma. Med Oncol. 2014;31(3):841. doi:10.1007/s12032-014-0374-0

21. Wang Y, Wang H, Jiang J, Cao X, Liu Q. Early decrease in postoperative serum albumin predicts severe complications in patients with colorectal cancer after curative laparoscopic surgery. World J Surg Oncol. 2018;16(1):192. doi:10.1186/ s12957-018-1493-4

22. Aminian A, Karimian F, Mirsharifi R, et al. Correlation of serum alkaline phosphatase with clinicopathological characteristics of patients with oesophageal cancer. East Mediterr Health J. 2011;17 (11):862-866.

23. Hung HY, Chen JS, Chien Y, et al. Pretreatment alkaline phosphatase elevation was associated with poor survival in colorectal cancer patients. Int J Colorectal Dis. 2017;32(12):1775-1778. doi:10.1007/ s00384-017-2907-4

24. Jin Y, Yuan MQ, Chen JQ, Zhang YP. Serum alkaline phosphatase predicts survival outcomes in patients with skeletal metastatic nasopharyngeal carcinoma. Clinics (Sao Paulo). 2015;70(4):264-272. doi:10.6061/clinics

25. Hossain DMS, Javaid S, Cai M, et al. Dinaciclib induces immunogenic cell death and enhances anti-PD1-mediated tumor suppression. J Clin Invest. 2018;128(2):644-654. doi:10.1172/JCI94586

The manuscript management system is completely online and includes a very quick and fair peer-review system, which is all easy to use. Visit http://www.dovepress.com/testimonials.php to read real quotes from published authors. 